\section{Hymenosides A-F, Six New Hemiterpene Glucosides from the Japanese Fern Hymenophyllum barbatum}

\author{
Yasushi Oiso, Masao ToYota, and Yoshinori AsaKawA* \\ Faculty of Pharmaceutical Sciences, Tokushima Bunri University, \\ Yamashiro-cho, Tokushima 770-8514, Japan. \\ Received October 26, 2000; accepted November 25, 2000
}

In the course of investigation of the bitter-tasting substances of the Japanese fern Hymenophyllum barbatum belonging to the family Hymenophyllaceae, six new hemiterpene glucosides called hymenosides $A-F(1-6)$ have been isolated from the methanol extract, together with an acyclic bis-bibenzyl derivative, perrottetin $\mathbf{H}$. This paper deals with the structure elucidation of the newly isolated glucosides.

Key words hemiterpene; glucoside; hymenoside; fern; Hymenophyllum barbatum

Little attention has been paid to the glycoside constituents of pteridophytes, although various types of triterpenoids and flavonoids have been isolated and their structures established. ${ }^{1)}$ We focus on the bitter- and hot-tasting substances of bryophytes and pteridophytes since these substance show interesting biological activities. ${ }^{2,3)}$ The Japanese fern Hymenophyllum barbatum (v. d. B.) Baker contains very bitter principles. Previously a nonbitter flavone, apigenin, has been isolated from this plant. ${ }^{4,5}$ Recently we reported the isolation and identification of an acyclic bis-bibenzyl, perrottetin $\mathrm{H}$, which is considered to be an important chemical fossil linking bryophytes and pteridophytes. ${ }^{\text {) }}$

H. barbatum was collected in August 1997, in Kaifu-gun, Tokushima. The air-dried and mechanically ground powder $(2.42 \mathrm{~kg})$ was extracted with methanol to give a crude extract $(459.2 \mathrm{~g})$. Part $(70.0 \mathrm{~g})$ of the ethyl acetate- soluble fraction $(119.6 \mathrm{~g})$ was chromatographed on silica gel and Sephadex LH-20, followed by preparative HPLC to yield six hemiterpene glucosides, hymenosides $\mathrm{A}-\mathrm{F}(\mathbf{1}-\mathbf{6})$, and perrottetin $\mathrm{H}$.

Hymenoside A (1), $[\alpha]_{\mathrm{D}}^{20}-21.8^{\circ}$, showed an $[\mathrm{M}+\mathrm{Na}]^{+}$at $\mathrm{m} / \mathrm{z} 555.1828$ in positive HR-FAB-MS, suggesting the molecular formula to be $\mathrm{C}_{27} \mathrm{H}_{32} \mathrm{O}_{11}$. The IR, UV, and ${ }^{13} \mathrm{C}-\mathrm{NMR}$ spectra of 1 indicated the presence of a benzene ring $(\lambda \mathrm{nm}$ [ $\log \varepsilon]: 201$ [4.64], 227 [4.15], 277 [3.53]), a hydroxyl $\left(3445 \mathrm{~cm}^{-1}\right)$, and two ester carbonyl groups $(1734,1242$, $1148 \mathrm{~cm}^{-1} ; \delta_{\mathrm{C}} 170.8$ [2'-O-C=O], 171.5 [3'-O-C=O]). The presence of five hydroxyl groups was confirmed by the formation of a pentaacetate. The ${ }^{1} \mathrm{H}-\mathrm{NMR}$ spectrum of $\mathbf{1}$ contained signals due to olefinic methyl $(\delta 1.62[\mathrm{~s}])$, two methylenes $(\delta 4.08,4.27$ [each brd, $J=12 \mathrm{~Hz}$ ] and $\delta 4.43$ [d, $J=$ $6.3 \mathrm{~Hz}])$ bearing an oxygen atom, two benzyl methylenes ( $\delta$ $3.57,3.61$ [each d, $J=15 \mathrm{~Hz}$ ], 3.65, 3.70 [each, d, $J=$ $15.7 \mathrm{~Hz}]$ ) bearing an ester carbonyl, a trisubstituted double bond ( $\delta 5.98[\mathrm{brt}, J=6.3 \mathrm{~Hz}]$ ), and two $p$-hydroxybenzene rings $(\delta 7.14,7.34$ [each, d, $J=8.2 \mathrm{~Hz}$ ], 7.10, 7.28 [each, d, $J=8.2 \mathrm{~Hz}]$ ). Acid hydrolysis of $\mathbf{1}$ gave D-glucopyranose which was confirmed by HPLC with an $[\alpha]_{\mathrm{D}}$ detector. Further purification of the reaction mixture afforded $p$-hydroxyphenylacetic acid of which the ${ }^{1} \mathrm{H}-\mathrm{NMR}$ spectrum was iden- tical to that of standard sample. Thus compound 1 possessed a hemiterpene with a trisubstitued double bond and two $p$-hydroxyphenylacetates. The $\beta$ configuration at $\mathrm{C}-1^{\prime}\left(\delta_{\mathrm{C}} 99.7\right)$ of glucose was determined by the coupling constant $(J=$ $8 \mathrm{~Hz})$ of an anomeric proton $(\delta 4.88)$. The position of each functional group was determined by HMBC and NOE spectra, as shown in Figs. 1 and 2 . The allylic carbon $(\delta 74.0)$ at $\mathrm{C}-1$, which was correlated with the olefinic methyl, was correlated with the anomeric proton $\left(\mathrm{C}-1^{\prime}\right)$. Furthermore the NOE was observed between the allylic methylene and the anomeric proton. The remaining two ester carbonyl carbons $(\delta 170.8$ and 171.5$)$ were correlated with the $\mathrm{C}-2^{\prime}(\delta 5.54$, br t, $J=8.8 \mathrm{~Hz})$ and C-3' $(\delta 5.83, \mathrm{t}, J=9.6 \mathrm{~Hz})$ of glucose, respectively. On the basis of the above spectral evidence, compound 1 was determined to be glucose with a hemiterpene at $\mathrm{C}-1^{\prime}$ and two $p$-substituted phenylacetates at $\mathrm{C}-2^{\prime}$ and $\mathrm{C}-3^{\prime}$. Moreover, NOEs were observed between the allylic methylene protons $(\mathrm{C}-1)$ and the methylene group at $\mathrm{C}-4$, indicating the $E$-configuration of the hemiterpene moiety. Thus the structure of hymenoside A was established to be $\mathbf{1}$.

Hymenoside $\mathrm{C}(\mathbf{3}),[\alpha]_{\mathrm{D}}^{21}-26.1^{\circ}$, had the molecular formula $\mathrm{C}_{27} \mathrm{H}_{32} \mathrm{O}_{12} \mathrm{Na}$ (positive HR-FAB-MS m/z 571.1817 $\left.[\mathrm{M}+\mathrm{Na}]^{+}\right)$. Comparison of the IR $(1734,1256$ [ester carbonyl], $1613 \mathrm{~cm}^{-1}$ [double bond]), UV ( $\lambda_{\max } \mathrm{nm}[\log \varepsilon]: 204$ [4.53], 283 [3.69]: benzene ring), ${ }^{1} \mathrm{H}-$, and ${ }^{13} \mathrm{C}-\mathrm{NMR}$ spectra with those of $\mathbf{1}$ showed that $\mathbf{3}$ differs structurally from $\mathbf{1}$ only in an additional hydroxyl group on one of the two benzene rings, although the same sugar unit and hemiterpene are affixed at $\mathrm{C}-1^{\prime}$. This was further confirmed by the formation of a hexaacetate by acetylation of $\mathbf{3}$. Thus compound 3 possessed 3,4-dihydroxyphenylacetoxyl $\left(\delta_{\mathrm{C}} 170.8\right.$ [2'-O-C $\left.=\mathrm{O}\right]$;

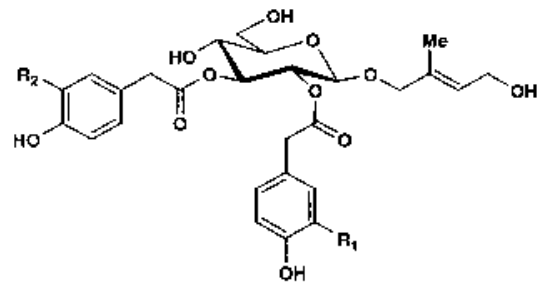

Hynceroside $A(I): R_{1}=R_{2}=H$ Hymenoside B (2): $\mathrm{R}_{1}-\mathrm{R}_{-2}-\mathrm{OH}$ Hytuenchide $C$ ( 3 ): $\mathrm{K}_{1}=\mathrm{OH}, \mathrm{R}_{2}=\mathrm{H}$ Hynenoside $D(4): R_{1} \cdot H_{1} R_{2}$. OII

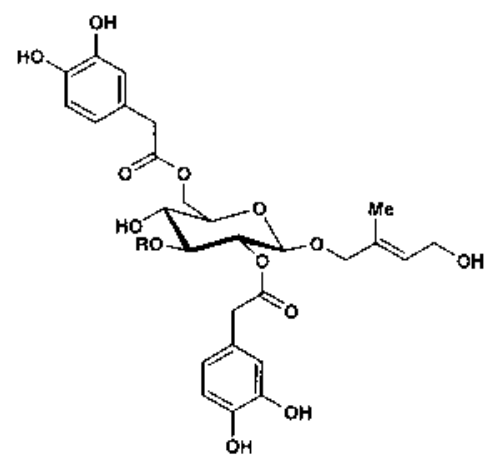

Hyminoside $\mathrm{H}$ (5): $\mathrm{R}=\mathrm{H}$

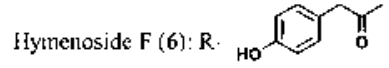




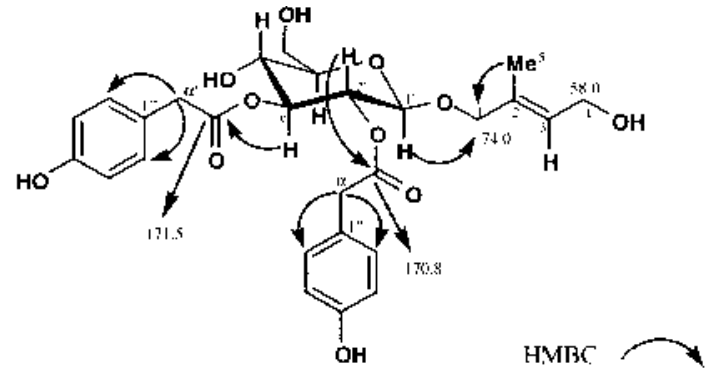

Fig. 1. HMBC Correlation of Hymenoside A (1)

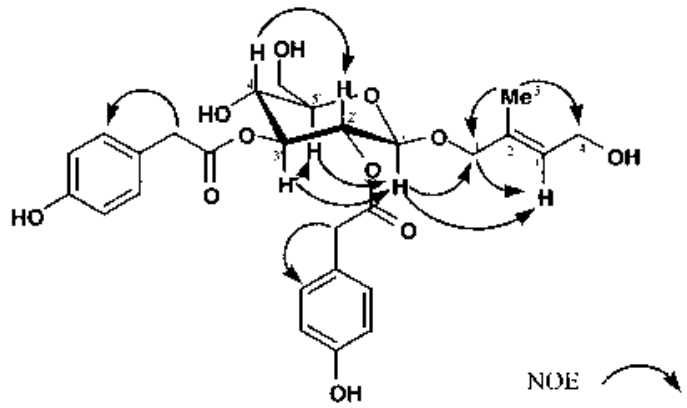

Fig. 2. NOE Correlation of Hymenoside A (1)

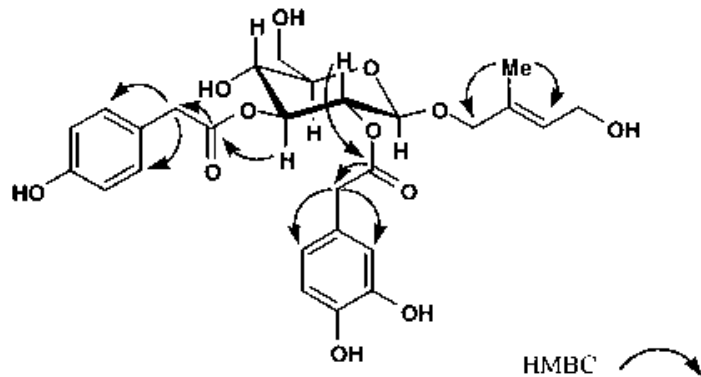

Fig. 3. HMBC Correlation of Hymenoside C (3)

$\left.\left[\delta_{\mathrm{H}-2^{\prime}} 5.58, \mathrm{dd}, J=10,8 \mathrm{~Hz}\right]\right)$ and $p$-hydroxyphenylacetoxyl $\left(\delta_{\mathrm{C}} 171.5\left[3^{\prime}-\mathrm{O}-\mathrm{C}=\mathrm{O}\right] ;\left[\delta_{\mathrm{H}-3^{\prime}} 5.86, \mathrm{t}, J=10 \mathrm{~Hz}\right]\right)$ groups. Each position was determined by the same HMBC (Fig. 3) and NOE techniques as carried out in $\mathbf{1}$.

The structures of the remaining glucosides $(\mathbf{2}, \mathbf{4}-\mathbf{6})$ were determined by careful analysis of 2D spectral data including HMBC and NOEs of each glucoside and their peracetates.

The presence of hemiterpene glucosides is very rare in nature. Cinnamic, caffeic, and benzoic glucosides are well known. ${ }^{7)}$ However, phenylacetic glucosides are also rare as natural products. Moreover all the glucosides isolated had a relatively strong bitter taste. This taste might be responsible for the presence of phenylacetate. ${ }^{8)}$

Acknowledgments This work was supported in part by a grant from the Sasagawa Foundation (to Y. O.). We thank Professor Shigeru Daigobou (Joetsu University of Education, Joetsu, Niigata, Japan) for the identification of fern species.

\section{References and Notes}

1) Murakami T., Tanaka N., "Progress in the Chemistry of Organic Natural Products," Vol. 54, ed. by Herz W., Grisebach H., Kirby G. W., Tamm Ch., Springer, Vienna, 1988, pp. 1-329.

2) Asakawa Y., "Progress in the Chemistry of Organic Natural Products," Vol. 42, ed. by Herz W., Grisebach H., Kirby G. W., Springer, Vienna,
1982, pp. 1-285.

3) Asakawa Y., "Progress in the Chemistry of Organic Natural Products," Vol. 65, ed. by Herz W., Kirby G. W., Moore R. E., Steglich W., Tamm Ch., Springer, Vienna, 1995, pp. 1-562.

4) Harada T., Saiki Y., Chem. Pharm. Bull., 3, 469-472 (1955).

5) Markham K. R., Wallace J. W., Phytochemistry, 19, 415-420 (1980).

6) Oiso Y., Toyota M., Asakawa Y., Chem. Pharm. Bull., 47, 297-298 (1999).

7) Shimomura H., Sashida Y., Adachi T., Phytochemistry, 26, 249-251 (1987).

8) Morishita T., Mukogawa Joshi Daigaku Kiyo, Shokumotsu-hen, 27, 39-43 (1980).

9) Hymenoside A (1): oil, positive HR-FAB-MS $m / z$ : $555.1828[\mathrm{M}+$ $\mathrm{Na}]^{+}$(Calcd for $\mathrm{C}_{27} \mathrm{H}_{32} \mathrm{O}_{11} \mathrm{Na}$ : 555.1842). $[\alpha]_{\mathrm{D}}^{20}-21.8^{\circ} \quad(c=4.8$, $\mathrm{MeOH}$ ). ${ }^{1} \mathrm{H}-\mathrm{NMR}$ (Pyridine- $d_{5}$ ): $\delta 1.62(3 \mathrm{H}, \mathrm{s}, \mathrm{H}-5), 3.57,3.61$ (each, $1 \mathrm{H}, \mathrm{d}, J=15 \mathrm{~Hz}, \mathrm{H}-\alpha^{\prime}$ ), 3.65, 3.70 (each, $1 \mathrm{H}, J=15.7 \mathrm{~Hz}, \mathrm{H}-\alpha$ ), 3.86 (1H, ddd, $J=9.6,4.6,2 \mathrm{~Hz}, \mathrm{H}-5^{\prime}$ ), 4.08, 4.27 (each, $1 \mathrm{H}$, br d, $J=12 \mathrm{~Hz}$, H-1), 4.29 (1H, dd, $\left.J=13,4.6 \mathrm{~Hz}, \mathrm{H}-6^{\prime}\right), 4.33\left(1 \mathrm{H}, \mathrm{t}, J=9.6 \mathrm{~Hz}, \mathrm{H}-4^{\prime}\right)$, $4.41\left(1 \mathrm{H}, \mathrm{dd}, J=13,2 \mathrm{~Hz}, \mathrm{H}-6^{\prime}\right), 4.43(2 \mathrm{H}, \mathrm{d}, J=6.3 \mathrm{~Hz}, \mathrm{H}-4), 4.88$ $\left(1 \mathrm{H}, \mathrm{d}, J=8 \mathrm{~Hz}, \mathrm{H}-1^{\prime}\right), 5.54\left(1 \mathrm{H}, \mathrm{br} \mathrm{t}, J=8.8 \mathrm{~Hz}, \mathrm{H}-2^{\prime}\right), 5.83(1 \mathrm{H}, \mathrm{t}, J=$ $\left.9.6 \mathrm{~Hz}, \mathrm{H}-3^{\prime}\right), 5.98(1 \mathrm{H}$, br t, $J=6.3 \mathrm{~Hz}, \mathrm{H}-3), 7.10(2 \mathrm{H}, \mathrm{d}, J=8 \mathrm{~Hz}, \mathrm{H}-$ $\left.3^{\prime \prime \prime}, 5^{\prime \prime \prime}\right), 7.14\left(2 \mathrm{H}, \mathrm{d}, J=8 \mathrm{~Hz}, \mathrm{H}-3^{\prime \prime}, 5^{\prime \prime}\right), 7.28\left(2 \mathrm{H}, \mathrm{d}, J=8 \mathrm{~Hz}, \mathrm{H}-2^{\prime \prime \prime}\right.$, $\left.6^{\prime \prime \prime}\right), 7.34\left(2 \mathrm{H}, \mathrm{d}, J=8 \mathrm{~Hz}, \mathrm{H}-2^{\prime \prime}, 6^{\prime \prime}\right) .{ }^{13} \mathrm{C}-\mathrm{NMR}\left(\mathrm{Py}-d_{5}\right): \delta 13.4$ (q, C-5), 39.9, $40.0\left(\mathrm{t}, \mathrm{C}-\alpha, \alpha^{\prime}\right), 58.0(\mathrm{t}, \mathrm{C}-4), 61.1\left(\mathrm{t}, \mathrm{C}-6^{\prime}\right), 68.4\left(\mathrm{~d}, \mathrm{C}-4^{\prime}\right), 72.4$ (d, C-2'), 74.0 (t, C-1), 76.3 (d, C-3'), 77.8 (d, C-5'), 99.7 (d, C-1'), $115.8,115.8$ (d, C-3", 3"', 5", 5"') $124.4,124.5$ (s, C-1"1, $\left.1^{\prime \prime \prime}\right), 128.7$ (d, C-3), 130.6, 130.7 (d, C-2", 2"', 6", 6"'), 132.2 (s, C-2), 157.4, 157.5 (s, $\left.\mathrm{C}-4^{\prime \prime}, 4^{\prime \prime \prime}\right), 170.8\left(\mathrm{~s}, 2^{\prime}-\mathrm{O}-\mathrm{C}=\mathrm{O}\right), 171.5\left(\mathrm{~s}, 3^{\prime}-\mathrm{O}-\mathrm{C}=\mathrm{O}\right)$.

Hymenoside B (2): oil, positive HR-FAB-MS $m / z$ : 587.1743 [M+ $\mathrm{Na}^{+}\left(\mathrm{Calcd}\right.$ for $\left.\mathrm{C}_{27} \mathrm{H}_{32} \mathrm{O}_{13} \mathrm{Na}: 587.1740\right)$. $[\alpha]_{\mathrm{D}}^{17}-24.0^{\circ}(c=2.92$, $\mathrm{MeOH})$. IR $(\mathrm{KBr}) \mathrm{cm}^{-1}: 3412,1734,1607,1364,1285$. UV $\lambda_{\max }$ EtOH nm $(\log \varepsilon): 284$ (3.44), 205 (4.31). ${ }^{1} \mathrm{H}-\mathrm{NMR}\left(\mathrm{Py}-d_{5}\right): \delta 1.63$ $(3 \mathrm{H}, \mathrm{s}, \mathrm{H}-5), 3.60,3.67\left(2 \mathrm{H}\right.$, each, d, $\left.J=15 \mathrm{~Hz}, \mathrm{H}-\alpha^{\prime}\right), 3.72(2 \mathrm{H}, \mathrm{s}, \mathrm{H}-$ $\alpha), 3.86\left(1 \mathrm{H}, \mathrm{m}, \mathrm{H}-5^{\prime}\right), 4.09,4.27$ (each, $1 \mathrm{H}$, br d, $\left.J=12 \mathrm{~Hz}, \mathrm{H}-1\right)$, $4.31\left(1 \mathrm{H}, \mathrm{dd}, J=12,5 \mathrm{~Hz}, \mathrm{H}-6^{\prime}\right), 4.32,\left(1 \mathrm{H}, \mathrm{t}, J=10 \mathrm{~Hz}, \mathrm{H}-4^{\prime}\right), 4.42$ $\left(1 \mathrm{H}, \mathrm{dd}, J=12,2 \mathrm{~Hz}, \mathrm{H}-6^{\prime}\right), 4.44(2 \mathrm{H}, \mathrm{d}, J=6 \mathrm{~Hz}, \mathrm{H}-4), 4.87(1 \mathrm{H}, \mathrm{d}$, $\left.J=8 \mathrm{~Hz}, \mathrm{H}-1^{\prime}\right), 5.57\left(1 \mathrm{H}, \mathrm{dd}, J=10,8 \mathrm{~Hz}, \mathrm{H}-2^{\prime}\right), 5.89(1 \mathrm{H}, \mathrm{t}, J=10 \mathrm{~Hz}$, H-3'), $6.00(1 \mathrm{H}$, br t, $J=6 \mathrm{~Hz}, \mathrm{H}-3), 6.89\left(1 \mathrm{H}, \mathrm{dd}, J=8,2 \mathrm{~Hz}, \mathrm{H}-6^{\prime \prime \prime}\right)$, $6.94\left(1 \mathrm{H}, \mathrm{dd}, J=8,2 \mathrm{~Hz}, \mathrm{H}-6^{\prime \prime}\right), 7.17\left(1 \mathrm{H}, \mathrm{d}, J=8 \mathrm{~Hz}, \mathrm{H}-5^{\prime \prime \prime}\right), 7.20(1 \mathrm{H}$, d, $\left.J=8 \mathrm{~Hz}, \mathrm{H}-5^{\prime \prime}\right), 7.28\left(1 \mathrm{H}, \mathrm{d}, J=2 \mathrm{~Hz}, \mathrm{H}-2^{\prime \prime \prime}\right), 7.35(1 \mathrm{H}, \mathrm{d}, J=2 \mathrm{~Hz}$, $\left.\mathrm{H}-2^{\prime \prime}\right) .{ }^{13} \mathrm{C}-\mathrm{NMR}\left(\mathrm{Py}-d_{5}\right): \delta 13.3$ (q, C-5), $40.2(\mathrm{t}, \mathrm{C}-\alpha), 40.3\left(\mathrm{t}, \mathrm{C}-\alpha^{\prime}\right)$, 57.9 (t, C-4), $61.1\left(\mathrm{t}, \mathrm{C}-6^{\prime}\right), 68.3$ (d, C-4'), $72.3\left(\mathrm{~d}, \mathrm{C}-2^{\prime}\right), 73.9$ (t, C-1), 76.1 (d, C-3'), 77.9 (d, C-5'), 99.7 (d, C-1'), 116.0, (d, C-5", 5'"), 117.3, 117.4 (d, C-2", C-2"'), 120.5, 120.7 (d, C-6", 6"') $125.3,125.4$ (s, C-1", 1"'), 128.6 (s, C-3), 145.8 (s, C-3", 3"'), 146.6, 146.7 (s, C-4", $\left.4^{\prime \prime \prime}\right), 170.8\left(\mathrm{~s}, 2^{\prime}-\mathrm{O}-\mathrm{C}=\mathrm{O}\right), 171.5\left(\mathrm{~s}, 3^{\prime}-\mathrm{O}-\mathrm{C}=\mathrm{O}\right)$.

Hymenoside C (3): oil, positive HR-FAB-MS m/z: 571.1817 [M+ $\mathrm{Na}]^{+}$(Calcd for $\mathrm{C}_{27} \mathrm{H}_{32} \mathrm{O}_{12} \mathrm{Na}$ : 571.1791). $[\alpha]_{\mathrm{D}}^{21}-26.1^{\circ}(c=2.38$, $\mathrm{MeOH})$. IR (KBr) cm ${ }^{-1}: 3412,1734,1613,1356,1256 .{ }^{1} \mathrm{H}-\mathrm{NMR}$ (Py$\left.d_{5}\right): \delta 1.64(3 \mathrm{H}, \mathrm{s}, \mathrm{H}-5), 3.60,3.66\left(\right.$ each, $\left.1 \mathrm{H}, \mathrm{d}, J=15 \mathrm{~Hz}, \mathrm{H}-\alpha^{\prime}\right)$, 3.68, 3.72 (each, $1 \mathrm{H}, \mathrm{d}, J=15 \mathrm{~Hz}, \mathrm{H}-\alpha$ ), $3.87(1 \mathrm{H}, \mathrm{ddd}, J=10,5,2 \mathrm{~Hz}$, H-5') 4.10, 4.28 (each, $1 \mathrm{H}$, br d, $J=12 \mathrm{~Hz}, \mathrm{H}-1), 4.32$ (1H, dd, $J=12$, $\left.5 \mathrm{~Hz}, \mathrm{H}-6^{\prime}\right), 4.35\left(1 \mathrm{H}, \mathrm{t}, J=10 \mathrm{~Hz}, \mathrm{H}-4^{\prime}\right), 4.43(1 \mathrm{H}, \mathrm{dd}, J=12,2 \mathrm{~Hz}, \mathrm{H}-$ $\left.6^{\prime}\right), 4.44(2 \mathrm{H}$, br d, $J=6 \mathrm{~Hz}, \mathrm{H}-4), 4.89\left(1 \mathrm{H}, \mathrm{d}, J=8 \mathrm{~Hz}, \mathrm{H}-1^{\prime}\right), 5.58$ $\left(1 \mathrm{H}, \mathrm{dd}, J=10,8 \mathrm{~Hz}, \mathrm{H}-2^{\prime}\right), 5.86\left(1 \mathrm{H}, \mathrm{t}, J=10 \mathrm{~Hz}, \mathrm{H}-3^{\prime}\right), 6.01(1 \mathrm{H}$, br dt, $J=6,1 \mathrm{~Hz}, \mathrm{H}-3), 6.94\left(1 \mathrm{H}, \mathrm{dd}, J=8,2 \mathrm{~Hz}, \mathrm{H}-6^{\prime \prime}\right), 7.12(2 \mathrm{H}, \mathrm{d}, J=$ $\left.8.5 \mathrm{~Hz}, \mathrm{H}-3^{\prime \prime \prime}, 5^{\prime \prime \prime}\right), 7.22\left(1 \mathrm{H}, \mathrm{d}, J=8 \mathrm{~Hz}, \mathrm{H}-5^{\prime \prime}\right), 7.31(2 \mathrm{H}, \mathrm{d}, J=8.5 \mathrm{~Hz}$, H-2'", $\left.6^{\prime \prime \prime}\right), 7.38\left(1 \mathrm{H}, \mathrm{d}, J=2 \mathrm{~Hz}, \mathrm{H}-2^{\prime \prime}\right) .{ }^{13} \mathrm{C}-\mathrm{NMR}\left(\mathrm{Py}-d_{5}\right): \delta 13.3$ (q, C-5), 40.0 (t, C- $\left.\alpha^{\prime}\right), 40.2(\mathrm{t}, \mathrm{C}-\alpha), 57.9$ (t, C-4), 61.1 (t, C-6'), 68.3 (d, C-4'), 72.3 (d, C-2'), 73.9 (t, C-1), 76.2 (d, C-3'), 77.8 (d, C-5'), 99.7 (d, C-1'), 115.7 (d, C-3"', 5'"'), 116.0 (d, C-5"), 117.4 (d, C-2"), 120.6 (d, C-6"), 124.6 (s, C-1"'), 125.2 (s, C-1"), 128.6 (d, C-3), 130.5 (d, C-2"', 6"'), $132.2(\mathrm{~s}, \mathrm{C}-2), 145.8\left(\mathrm{~s}, \mathrm{C}-4^{\prime \prime}\right), 146.7\left(\mathrm{~s}, \mathrm{C}-3^{\prime \prime}\right), 157.4(\mathrm{~s}$, $\left.\mathrm{C}-4^{\prime \prime \prime}\right), 170.8\left(\mathrm{~s}, 2^{\prime}-\mathrm{O}-\mathrm{C}=\mathrm{O}\right), 171.5\left(\mathrm{~s}, 3^{\prime}-\mathrm{O}-\mathrm{C}=\mathrm{O}\right)$.

Hymenoside D (4): oil, positive HR-FAB-MS $m / z$ : 571.1769 [M+ $\mathrm{Na}]^{+}$(Calcd for $\mathrm{C}_{27} \mathrm{H}_{32} \mathrm{O}_{12} \mathrm{Na}$ : 571.1791). $[\alpha]_{\mathrm{D}}^{21}-20.0^{\circ}(c=1.18$, $\mathrm{MeOH})$. IR $(\mathrm{KBr}) \mathrm{cm}^{-1}: 3466,1734,1622,1362,1248$. UV $\lambda_{\max }$ $(\mathrm{EtOH}) \mathrm{nm}(\log \varepsilon): 283$ (3.70), 203 (4.62). ${ }^{1} \mathrm{H}-\mathrm{NMR}\left(\mathrm{Py}-d_{5}\right): \delta 1.64$ $(3 \mathrm{H}, \mathrm{s}, \mathrm{H}-5), 3.59,3.64$ (each, $\left.1 \mathrm{H}, \mathrm{d}, J=15 \mathrm{~Hz}, \mathrm{H}-\alpha^{\prime}\right), 3.72(2 \mathrm{H}, \mathrm{s}, \mathrm{H}-$ $\alpha$ ), $3.88\left(1 \mathrm{H}\right.$, ddd, $\left.J=10,5,2 \mathrm{~Hz}, \mathrm{H}-5^{\prime}\right), 4.10,4.29$ (each, $1 \mathrm{H}, \mathrm{br}$, $J=12 \mathrm{~Hz}, \mathrm{H}-1), 4.31\left(1 \mathrm{H}, \mathrm{dd}, J=12,5 \mathrm{~Hz}, \mathrm{H}-6^{\prime}\right), 4.34(1 \mathrm{H}, \mathrm{t}, J=$ $\left.10 \mathrm{~Hz}, \mathrm{H}-4^{\prime}\right), 4.43\left(1 \mathrm{H}, \mathrm{dd}, J=12,2 \mathrm{~Hz}, \mathrm{H}-6^{\prime}\right), 4.46(2 \mathrm{H}, \mathrm{d}, J=6 \mathrm{~Hz}$, $\mathrm{H}-4), 4.90\left(1 \mathrm{H}, \mathrm{d}, J=8 \mathrm{~Hz}, \mathrm{H}-1^{\prime}\right), 5.57\left(1 \mathrm{H}, \mathrm{dd}, J=10,8 \mathrm{~Hz}, \mathrm{H}-2^{\prime}\right)$, $5.87\left(1 \mathrm{H}, \mathrm{t}, J=10 \mathrm{~Hz}, \mathrm{H}-3^{\prime}\right), 6.01(1 \mathrm{H}, \mathrm{br} \mathrm{dt}, J=6,1 \mathrm{~Hz}, \mathrm{H}-3), 6.90$ 
(1H, dd, $\left.J=8,2 \mathrm{~Hz}, \mathrm{H}-6^{\prime \prime \prime}\right), 7.16\left(2 \mathrm{H}, \mathrm{d}, J=8.5 \mathrm{~Hz}, \mathrm{H}-3^{\prime \prime}, 5^{\prime \prime}\right), 7.19(1 \mathrm{H}$, d, $\left.J=8 \mathrm{~Hz}, \mathrm{H}-5^{\prime \prime \prime}\right), 7.35\left(1 \mathrm{H}, \mathrm{d}, J=2 \mathrm{~Hz}, \mathrm{H}-2^{\prime \prime}\right), 7.37(2 \mathrm{H}, \mathrm{d}, J=8.5 \mathrm{~Hz}$, $\left.\mathrm{H}-2^{\prime \prime}, 6^{\prime \prime}\right) .{ }^{13} \mathrm{C}-\mathrm{NMR}\left(\mathrm{Py}-d_{5}\right): \delta 13.3$ (q, C-5), 39.8 (t, C- $\alpha$ ), $40.3(\mathrm{t}, \mathrm{C}-$ $\left.\alpha^{\prime}\right), 57.9$ (t, C-4), 61.1 (t, C-6'), 68.2 (d, C-4'), 72.3 (d, C-2'), 73.9 (t, C-1), 76.1 (d, C-3'), 77.8 (d, C-5'), $99.6\left(\mathrm{~d}, \mathrm{C}-1^{\prime}\right), 115.7$ (d, C-3", 5"'), 115.9 (d, C-5"'), 117.3 (d, C-2"'), 120.4 (d, C-6"'), 124.4 (s, C-1"), 125.3 (s, C-1"'), 128.8 (d, C-3), 130.6 (d, C-2", 6"), 132.0 (s, C-2), 145.8 (s, C-4"'), 146.7 (s, C-3"'), 157.4 (s, C-4"), 170.7 (s, 2'-O-C= $\mathrm{O}), 171.5\left(\mathrm{~s}, 3^{\prime}-\mathrm{O}-\mathrm{C}=\mathrm{O}\right)$.

Hymenoside E (5): oil, positive HR-FAB-MS $m / z$ : 587.1733 [M+ $\mathrm{Na}^{+}$(Calcd for $\mathrm{C}_{27} \mathrm{H}_{32} \mathrm{O}_{13} \mathrm{Na}$ : 587.1741). $[\alpha]_{\mathrm{D}}^{22}-8.2^{\circ} \quad(c=0.66$, $\mathrm{MeOH})$. IR $(\mathrm{KBr}) \mathrm{cm}^{-1}: 3375,1730,1606,1361,1286$. UV $\lambda_{\text {max }}$ $(\mathrm{EtOH}) \mathrm{nm}(\log \varepsilon): 283$ (3.91), 204 (4.83). ${ }^{1} \mathrm{H}-\mathrm{NMR}\left(\mathrm{Py}-d_{5}\right): \delta 1.70$ $(3 \mathrm{H}, \mathrm{s}, \mathrm{H}-5), 3.69,3.74$ (each, $\left.1 \mathrm{H}, \mathrm{d}, J=15 \mathrm{~Hz}, \mathrm{H}-\alpha^{\prime}\right), 3.80,3.85$ (each, $1 \mathrm{H}, \mathrm{d}, J=15 \mathrm{~Hz}, \mathrm{H}-\alpha), 3.95\left(1 \mathrm{H}, \mathrm{ddd}, J=9,6,2 \mathrm{~Hz}, \mathrm{H}-5^{\prime}\right), 4.04$ $\left(1 \mathrm{H}, \mathrm{t}, J=9 \mathrm{~Hz}, \mathrm{H}-4^{\prime}\right), 4.19\left(1 \mathrm{H}, \mathrm{t}, J=9 \mathrm{~Hz}, \mathrm{H}-3^{\prime}\right), 4.19,4.37$ (each, $1 \mathrm{H}$, br d, $J=12 \mathrm{~Hz}, \mathrm{H}-1), 4.46(2 \mathrm{H}, \mathrm{d}, J=7 \mathrm{~Hz}, \mathrm{H}-4), 4.70(1 \mathrm{H}, \mathrm{dd}, J=$ $\left.12,6 \mathrm{~Hz}, \mathrm{H}-6^{\prime}\right), 4.84\left(1 \mathrm{H}, \mathrm{d}, J=8 \mathrm{~Hz}, \mathrm{H}-1^{\prime}\right), 4.98(1 \mathrm{H}, \mathrm{dd}, J=12,2 \mathrm{~Hz}$, $\left.\mathrm{H}-6^{\prime}\right), 5.64\left(1 \mathrm{H}, \mathrm{dd}, J=9,8 \mathrm{~Hz}, \mathrm{H}-2^{\prime}\right), 6.10(1 \mathrm{H}, \mathrm{brdt}, J=7,1 \mathrm{~Hz}, \mathrm{H}-$ 3), $6.91\left(1 \mathrm{H}, \mathrm{dd}, J=8,2 \mathrm{~Hz}, \mathrm{H}-6^{\prime \prime \prime}\right), 6.94\left(1 \mathrm{H}, \mathrm{dd}, J=8,2 \mathrm{~Hz}, \mathrm{H}-6^{\prime \prime}\right)$, $7.192\left(1 \mathrm{H}, \mathrm{d}, J=8 \mathrm{~Hz}, \mathrm{H}-5^{\prime \prime \prime}\right), 7.195\left(1 \mathrm{H}, \mathrm{d}, J=8 \mathrm{~Hz}, \mathrm{H}-5^{\prime \prime}\right), 7.39(1 \mathrm{H}$, d, $\left.J=2 \mathrm{~Hz}, \mathrm{H}-2^{\prime \prime \prime}\right), 7.40\left(1 \mathrm{H}, \mathrm{d}, J=2 \mathrm{~Hz}, \mathrm{H}-2^{\prime \prime}\right) .{ }^{13} \mathrm{C}-\mathrm{NMR}\left(\mathrm{Py}-d_{5}\right): \delta$ 14.0 (q, C-5), 40.8 (t, C- $\left.\alpha^{\prime}\right), 41.2$ (t, C- $\alpha$ ), 58.6 (t, C-4), 64.7 (t, C-6'), 71.7 (d, C-4'), 74.5 (t, C-1), 75.2(d, C-2'), $75.3\left(\mathrm{~d}, \mathrm{C}-5^{\prime}\right), 75.9$ (d, C$\left.3^{\prime}\right), 100.6\left(\mathrm{~d}, \mathrm{C}-1^{\prime}\right), 116.5,116.6\left(\mathrm{~d}, \mathrm{C}-5^{\prime \prime}, 5^{\prime \prime \prime}\right), 117.9\left(\mathrm{~d}, \mathrm{C}-2^{\prime \prime \prime}\right), 118.0$ (d, C-2"), 121.0 (d, C-6"'), 121.2 (d, C-6"), 126.1, 126.1 (s, C-1", 1"'), 129.3 (d, C-3), 133.0 (s, C-2), 146.3, 146.4 (s, C-4", 4"'), 147.2, 147.3 (s, C-3", $\left.3^{\prime \prime \prime}\right), 171.5\left(\mathrm{~s}, 2^{\prime}-\mathrm{O}-\mathrm{C}=\mathrm{O}\right), 172.3\left(\mathrm{~s}, 6^{\prime}-\mathrm{O}-\mathrm{C}=\mathrm{O}\right)$.

Hymenoside F (6): oil, positive HR-FAB-MS $m / z$ : $721.2117[\mathrm{M}+\mathrm{Na}]^{+}$ (Calcd for $\mathrm{C}_{35} \mathrm{H}_{38} \mathrm{O}_{15} \mathrm{Na}$ : 721.2108). $[\alpha]_{\mathrm{D}}^{20}-10.2^{\circ}(c=2.37, \mathrm{MeOH})$. IR $(\mathrm{KBr}) \mathrm{cm}^{-1}: 3285,1732,1608,1356,1287$. UV $\lambda_{\text {max }}(\mathrm{EtOH}) \mathrm{nm}$ $(\log \varepsilon): 283$ (3.85), 202 (4.88). ${ }^{1} \mathrm{H}-\mathrm{NMR}\left(\mathrm{Py}-d_{5}\right): \delta 1.62(3 \mathrm{H}, \mathrm{s}, \mathrm{H}-5)$, $3.55,3.61$ (each, $1 \mathrm{H}, \mathrm{d}, J=15 \mathrm{~Hz}, \mathrm{H}-\alpha^{\prime}$ ), 3.63, 3.67 (each, $1 \mathrm{H}, \mathrm{d}, J=$ $15 \mathrm{~Hz}, \mathrm{H}-\alpha), 3.65,3.70$ (each, $\left.1 \mathrm{H}, \mathrm{d}, J=15 \mathrm{~Hz}, \mathrm{H}-\alpha^{\prime \prime}\right), 3.93(1 \mathrm{H}$, ddd, $\left.J=10,6,2 \mathrm{~Hz}, \mathrm{H}-5^{\prime}\right), 4.08\left(1 \mathrm{H}, \mathrm{t}, J=10 \mathrm{~Hz}, \mathrm{H}-4^{\prime}\right), 4.12,4.27$ (each, $1 \mathrm{H}$, br d, $J=12 \mathrm{~Hz}, \mathrm{H}-1), 4.41(2 \mathrm{H}$, br dd, $J=6,2 \mathrm{~Hz}, \mathrm{H}-4), 4.62(1 \mathrm{H}$, dd, $\left.J=12,6 \mathrm{~Hz}, \mathrm{H}-6^{\prime}\right), 4.81\left(1 \mathrm{H}, \mathrm{d}, J=8 \mathrm{~Hz}, \mathrm{H}-1^{\prime}\right), 4.88(1 \mathrm{H}, \mathrm{dd}, J=$ $\left.12,2 \mathrm{~Hz}, \mathrm{H}-6^{\prime}\right), 5.53\left(1 \mathrm{H}, \mathrm{dd}, J=10,8 \mathrm{~Hz}, \mathrm{H}-2^{\prime}\right), 5.76(1 \mathrm{H}, \mathrm{t}, J=$ $\left.10 \mathrm{~Hz}, \mathrm{H}-3^{\prime}\right), 6.01(1 \mathrm{H}, \mathrm{brdt}, J=6,1 \mathrm{~Hz}, \mathrm{H}-3), 6.87(1 \mathrm{H}, \mathrm{dd}, J=8$, $\left.2 \mathrm{~Hz}, \mathrm{H}-6^{\prime \prime \prime \prime}\right), 6.90\left(1 \mathrm{H}, \mathrm{dd}, J=8,2 \mathrm{~Hz}, \mathrm{H}-6^{\prime \prime}\right), 7.08(2 \mathrm{H}, \mathrm{d}, J=8.5 \mathrm{~Hz}$, H-3"', $\left.5^{\prime \prime \prime}\right), 7.16\left(1 \mathrm{H}, \mathrm{d}, J=8 \mathrm{~Hz}, \mathrm{H}-5^{\prime \prime \prime \prime}\right), 7.18\left(1 \mathrm{H}, \mathrm{d}, J=8 \mathrm{~Hz}, \mathrm{H}-5^{\prime \prime}\right)$, $7.26\left(2 \mathrm{H}, \mathrm{d}, J=8.5 \mathrm{~Hz}, \mathrm{H}-2^{\prime \prime \prime}, 6^{\prime \prime \prime}\right), 7.31\left(1 \mathrm{H}, \mathrm{d}, J=2 \mathrm{~Hz}, \mathrm{H}-2^{\prime \prime \prime \prime}\right), 7.34$ $\left(1 \mathrm{H}, \mathrm{d}, J=2 \mathrm{~Hz}, \mathrm{H}-2^{\prime \prime}\right) .{ }^{13} \mathrm{C}-\mathrm{NMR}\left(\mathrm{Py}-d_{5}\right): \delta 13.3$ (q, C-5), 39.9 (t, C$\left.\alpha^{\prime}\right), 40.1,40.2\left(\mathrm{t}, \mathrm{C}-\alpha, \alpha^{\prime \prime}\right), 57.9$ (t, C-4), $63.5\left(\mathrm{t}, \mathrm{C}-6^{\prime}\right), 68.6\left(\mathrm{~d}, \mathrm{C}-4^{\prime}\right)$, 72.0 (d, C-2'), 74.0 (t, C-1), 74.4 (d, C-5'), 75.8 (d, C-3'), 99.4 (d, C$\left.1^{\prime}\right), 115.7$ (d, C-3"', 5"') $), 115.9,116.0\left(\mathrm{~d}, \mathrm{C}-5^{\prime \prime}, 5^{\prime \prime \prime}\right), 117.25,117.35$ (d, C-6", 6"'"'), 120.4, 120.6 (d, C-2", 2"'"'), 124.4 (s, C-1"'), 125.1, 125.4 (s, C-1", $\left.1^{\prime \prime \prime \prime}\right), 129.0$ (d, C-3), 130.5 (d, C-2"', 6"'), 132.0 (s, C-2), 145.75, 145.83 (s, C-4", 4'"'), 146.7 (s, C-3", C-3"'"'), 157.4 (s, C-4"'), 170.7 (s, $\left.2^{\prime}-\mathrm{O}-\mathrm{C}=\mathrm{O}\right), 171.3\left(\mathrm{~s}, 3^{\prime}-\mathrm{O}-\mathrm{C}=\mathrm{O}\right), 171.6\left(\mathrm{~s}, 6^{\prime}-\mathrm{O}-\mathrm{C}=\mathrm{O}\right)$. 